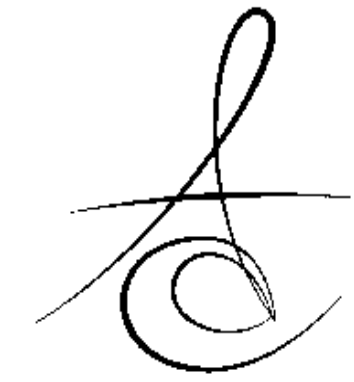

Makale Kodu/Article code: 2253

Makale Gönderilme tarihi: 23.04 .2015

Kabul Tarihi: 02.06.2015

\section{KİŞİYE ÖZGÜ DENTAL İMPLANTLAR: DERLEME}

\author{
CUSTOM-MADE DENTAL IMPLANTS : REVIEW
}

Dt. Yavuz ALTINTOP**

Prof. Dr. Bora BAĞIŞ**

\section{öz}

Hastaların dental implantlara olan ilgisinin artmasıyla beraber dental implantolojide de her geçen gün yeni gelişmeler olmaktadır. Son zamanlarda hastalara çekim sonrası immediat olarak yerleştirilebilen; hastanın kaybettiği dişlerinin kökleri ile aynı şekilde; kişiye özgü olarak hazırlanan implantlar kullanılmaktadır. Yapılan bu derlemede, zirkonyadan ve titanyumdan hazırlanan kişiye özgü implantların üretim prosedürleri hakkında bilgi verilmiştir.

Anahtar kelimeler: Kişiye özgü implant, Lazer sinterleme, CADCAM

Dental implantlar, kaybedilen dişlerin hastaya geri kazandırılması için uygulanan başarılı bir tedavi yöntemi olarak kabul edilmektedir. 10 yıllık hasta takibi sonucunda \%90-100 lük bir başarı oranına sahip olduğu rapor edilmektedir. ${ }^{1}$ İmplant kullanımının son yıllardaki artışına karşın implantların ne materyalinde ne de formunda önemli bir değişiklik olmamıştır. ${ }^{2}$

Dental implant tedavisinde, başlangıçta, diş çekimi ve implantların yerleştirilmesi arasında 6-9 aylık iyileşme periyodu olması önerilmiştir. Daha sonra, bu süre 2-3 ay indirilerek daha erken implant yerleştirme önerilmiştir. Ancak son dönemde, hastaların estetik ve fonksiyonel beklentilerinin artması ile bazı iyi seçilmiş vakalarda, diş çekimini takiben birkaç gün içinde hatta dişler çekilir çekilmez immediat implantasyon uygulanmaya başlanmıştır. ${ }^{3}$ Dental implant tedavisinde gelişim daha çok, immediat implant yerleştirmek; klinik prosedürleri basitleştirmek ve tedavi süresini kısaltmak yolunda ilerlemektedir. ${ }^{4,5}$

Diş çekimi ve implantasyon arasındaki sürenin kısa olduğu vakalardaki sonuçlar ile geç implantasyon vakalarındaki sonuçların benzer olduğu görülmüştür. ${ }^{2}$

\section{ABSTRACT}

There have been new progresses with every passing day in dental implantology as well as the patients' increasing interests to dental implants. Lately, implants which can be placed immediately after the tooth extractions and which can be prepared as the same shapes of the roots that the patients lost, are used. In this review, information has been given about the production of custom-made, root-analogue zirconia and titanium implants.

Key words: Custom-made implant, Laser sintering, CADCAM

İmmediat implant yerleştirmenin en büyük avantajı tedavi süresinin kısalması ve cerrahi girişimlerin sayısının azaltılmasıdır. Bu sayede hastanın yaşam kalitesi artmakta ve tedavi masrafları azalmaktadır. $^{2}$

Çekim sonrası immediat implant yerleştirme işleminde, çekim soketi ve endoosseoz implant şekli arasındaki uyumsuzluk büyük bir sorun olarak karşımıza çıkar. Diş çekimini takiben çekim soketi silindir tipli bir implanttan çok daha büyük bir çapa sahiptir, bu da implant yerleştirme işleminde ilave kemik grefti ve membran kullanımı gereksinimini ortaya çıkarabilir. ${ }^{4,5}$

Kemik grefti ve membran kullanımı cerrahi prosedürü zorlaştırmaktadır, ayrıca çekim soketini prefabrike implanta uygun hale getirmek için de kemik frezlerinin kullanılması gerekmektedir. Ancak çekilen dişle aynı morfolojiye sahip kişiye özgü bir implantın çekim soketine yerleştirilmesi ile implant ve soket arasındaki şekil uyumsuzluğu ortadan kaldırılabilir dolayısıyla kemik grefti, membran ve kemik frezlerinin kullanılmasına da gerek kalmayacaktır ve bu sayede cerrahi prosedür de kolaylaşabilir.

* Ankara Üniversitesi Diş Hekimliği Fakültesi Periodontology AD

**İzmir Katip Çelebi Üniversitesi Diş Hekimliği Fakültesi Protetik Diş Tedavisi AD 
Bu nedenle ilk olarak Hodosh ve $\operatorname{ark}^{6}$ tarafından 1969 yılında çekilen dişin köküyle aynı morfolojiye sahip kişiye özgü implant üretilmiş ve çekim soketine kaybedilen dişin yerine yerleştirilmiştir. Hodosh ve $\mathrm{ark}^{6}$ tarafından yapılan implantın üretiminde polimetilmetakrilat kullanılmış ve osseointegre olmak yerine yumuşak doku tarafından sarılmıştır. Lundgren ve $a \mathrm{rk}^{7}$ 1992 yılında kök şekilli implant fikrini yeniden ortaya atmıştır. Ancak Lundgren ve $a^{7}{ }^{7}$ bu sefer implant materyali olarak polimer yerine titanyum kullanmışlar ve sonuç olarak \%88'lik osseointegrasyon elde etmişlerdir. Benzer şekilde Kohal ve $\operatorname{ark}^{8}$ tarafından yapılan kök şekilli titanyum implantların osteointegrasyonu ve klinik stabilitesini inceleyen deneysel çalışmada da olumlu sonuçlar elde edilmiştir.

Kök şekilli implant kullanımının avantajları olarak geleneksel kemik frezleri, kemik grefti ve membran kullanımına gerek kalmaması; implantasyona hazırlık kapsamında gereken diğer travmatik prosedürlerin eliminasyonu ve böylece de hastanın artan konforu gösterilebilir. Ayrıca kökün şekli birebir yansıtılarak daha estetik sonuçlar da elde edilmiş olacaktır. ${ }^{6-13}$

Bazı rezidüel kretler ise oldukça incedir ve standart çapta kök şekilli implantlar yerleştirildiğinde, implant çevresinde yaklaşık 1-2 mm'lik çevresel kemik kalmamaktadır. ${ }^{14}$ Rezorbe olmuş mandibular posterior bölgede standart çapta, kök şekilli implantların kullanılması oldukça güçtür. ${ }^{14}$

Böyle durumlarda ideal yaklaşım kemiğin horizontal ögmentasyonunun sağlanmasıdır. Ancak bu şekilde daha iyi estetik ve fonksiyonel sonuçlar elde edilir. İmplanta daha iyi kemik desteği sağlanır, alt ve üst çene arasındaki mesafe azaltılır. ${ }^{14,15}$ Ancak hastalar tarafından, kemiğin rekonstrükte edildiği cerrahi işlemler riskleri ve ekonomik nedenler yüzünden kabul edilmeyebilmektedir. ${ }^{14,15}$

Blade tip implantların 3 mm'ye kadar dar kemiklerde ve hem maksiller hem de mandibular kretlerde kullanılma endikasyonu olduğu söylenmiştir. ${ }^{16-22}$

Blade implantlar ince, düz ve genellikle dikdörtgen şekillidir, uzun kenarında ağız içine açılan ve sabit protezi destekleyen bir ya da iki adet çıkıntı vardır. ${ }^{16-22}$

Blade tip implantlar yıllarca kullanılmış ancak kök şekilli implantların kullanılması ile popülaritesi azalmıştır. Blade tip implantlar postoperatif komplikasyonlar, primer stabilitenin sağlanması ve yükleme protokollerinin zorluğu nedeniyle yüksek başarısızlık oranı göstermektedir. ${ }^{21-25}$ Bununla birlikte bazı blade implantlar 20-30 yıl başarılı şekilde ağızda kalmıştır ${ }^{20-22}$ ve bu implantlar ince posterior mandibulada kullanılabilmektedir. ${ }^{21,22}$ Mangano F. ve ark ${ }^{14}$ tarafından 2013 yılında blade tipte kişisel implantların üretilmesi ile ilgili bir prosedür tanıtılmıştır.

Günümüzde CAD/CAM ve hızlı prototipleme yöntemiyle daha öngörülebilir sonuçları olan daha az invaziv ve geçmişteki blade tip implantlara nazaran daha az teknik hassasiyet gerektiren kişiye özgü blade tipte implantlar üretilebilmektedir.

Osseointegrasyon canlı kemik dokusu ve yüklenmiş implant arasındaki yapısal ve fonksiyonel bağlantı olarak tanımlanmaktadır. İmplant stabilitesi için kritik önem taşıyan osseointegrasyon mekanizması, implantın yüklenmesi ve uzun dönem başarısı için ön koşuldur. ${ }^{26,27}$

İmplant yüzey özelliklerinin, osseointegrasyon mekanizmasını destekleyen en önemli faktörlerden biri olduğu kabul edildiğinden, çalışmalar osseointegrasyon mekanizmasını optimize etmeye odaklanmış ve implant yüzey modifikasyonları geniş oranda incelemiştir. ${ }^{28,29}$

Günümüzde, dental implantlar titanyum çubukların mekanik olarak işlenmesi ve bunu takiben, kum$\operatorname{lama}^{28}$, asit ${ }^{30,31}$, veya anodizasyon ile pürüzlendir$\mathrm{me}^{32,33}$, implant yüzeylerinin CaP ile kaplanması ${ }^{34}$, kimyasal modifikasyonlar ${ }^{28,29,32}$ gibi işlemlerle implant yüzey dizaynının modifiye edilmesi ile üretilir.

Üretim tekniklerindeki limitasyonlar nedeniyle açık poröz yapıların oluşmasını engellenmesine rağmen, ${ }^{35,36}$ porözitenin, por genişlikleri ile dağılımlarının ve mekanik özelliklerin kontrol edilebildiği poröz yapıya sahip implantların üretilmesi için talep vardır. ${ }^{37-40}$

Katmanlı üretim (additive manufacturing) metotları bu durumun üstesinden gelebilmek için önerilmiştir. 37-41

Katı serbest şekilli fabrikasyonu (solid freeform fabrication), tabakalı üretim (layered manufacturing) veya doğrudan dijital üretim (direct digital manufacturing) olarak da bilinen katmanlı üretim (additive manufacturing), üç boyutlu sanal model verileri doğrultusunda yapısı ve şeklinin tanımlanması ile direkt olarak fiziksel objeleri oluşturmak için kullanılan bir stratejidir. ${ }^{39-41}$

Özellikle katmanlı üretim, bilgisayar destekli tasarım (CAD) verilerinden veya bilgisayar destekli tıbbi görüntüleme teknolojilerinden sağlanan katmankatman şeklindeki verilerden, direkt olarak fiziksel modeli oluşturabilen teknikleri içerir. ${ }^{39-42}$ 
Direkt metal lazer sinterleme (DMLS), toz metaller, radyant Isitıcılar ve bilgisayar kontrollü lazer ile bir objenin tabaka tabaka oluşturulmasını sağlayan lazer destekli katmanlı üretim tekniğidir. ${ }^{39-43}$

Temelde, makine, materyalin katmanlarını tabakalama tekniği ile uygulayarak hareketli platform üzerinde objenin üretimini sağlar. ${ }^{39-43}$

Her bir tabaka için, makine doğru kalınlığa sahip $(0.1 \mathrm{~mm})$ toz tabakasını ortaya koymaktadır. Daha sonra, yüksek güce sahip lazer ışını bu toz yatağı üzerine yönlendirilir ve bilgisayar destekli tasarım dosyasına uygun olarak odak bölgesinde bulunan metal tozlarını kaynaşıımak için programlanır. Böylelikle ince bir metal tabakası oluşturulur. Platform önceden programlanmış tabaka kalınlığını aşağı hareket ettirir, taze toz tabakası ortaya konur ve daha sonra lazer ekspozürü ile sonraki tabaka eritilir böylece önceki tabakaya uyum sağlar. Bu süreç, obje üretilinceye kadar, tabaka tabaka devam eder. ${ }^{39-43}$

Direkt metal lazer sinterleme, her bir tabakadaki porözitenin ve aynı zamanda por bağlantısııın, boyutun, şeklin ve dağılımın ve buna bağlı olarak implantın üç boyutlu yapısının kontrolünün sağlanmasını imkanlı hale getirir. Bu durum lazer gücü ve pik gücü, lazer nokta çapı, katman kalınlı̆ı, tarama mesafesi, tarama hızı ve tarama stratejisi veya orjinal titanyum partikül boyutunun modifiye edilmesi gibi parametrelerin değiştirilmesi ile sağlanır. ${ }^{39-43}$

Bu tekniğin en önemli avantajı, açık porların oluşturduğu bağlantıların yüksek seviyesinin, kemik dokusunun içeri doğru büyümesine ve vaskülarizasyona izin vermesidir. Böylece implantın uzun süreli güvenilirliği için temel faktör olan osseointegrasyonun arttırılması sağlanır. ${ }^{39-43}$ Buna ek olarak, biyomateryallerin mekanik özelliklerinin mikroyapılarına bağı olması nedeniyle, direkt lazer sinterleme kemiğe yakın mekanik özellikleri (sertlik) olan poröz implantların üretilmesinde kullanılabilmektedir. ${ }^{39-43}$

Aslında tamamıyla yoğun komponentle karşlaştırılacak olursa poröz implantlar azalmış gerilme direnci ve elastik modülüne sahiptirler. Sonuç olarak kemik dokusunun elastik modülü ve gerilme direnci ile daha iyi uyum sağlayacak şekilde, poröz materyalin mekanik özellikleri uygun hale getirilebilir. Bu nedenle kemikimplant elastik modülü uyumsuzluğundan kaynaklanan stres bölgeleri gibi istenmeyen durumlar ortadan kaldırılır. ${ }^{39-43}$

Direkt metal lazer sinterlemenin diğer bir avantajı ise, zaman-maliyet rekabeti içerisinde, komp- leks şekilli kişiye özgü(custom-made) titanyum implantların üretilmesi için sınırsız özgürlüğe ve yeteneğe sahip olmasıdır. ${ }^{42,43}$

Direkt metal lazer sinterleme, doğrudan bilgisayar destekli tasarım (CAD) verilerinden parçaları bir araya getirir. Başka malzemeye intiyaç duyulmaz. Sonuçta kesme ve milleme sürecinin aksine, direkt metal lazer sinterleme teknolojisi ile daha az israf oluştur ve neredeyse materyal kaybı hiç yoktur. ${ }^{42,43}$

Henüz litaratürde direkt metal lazer sinterleme ile üretilen titanyum implantlar hakkında yeterli bilgi olmamasına rağmen, katmanlı üretim ve direkt metal lazer sinterleme teknolojileri ile implant ve biyomateryal üretimi kabul görmüştür.

Titanyum kanıtlanmış biyouyumluluğu ve mekanik özellikleri nedeniyle son 40 yılda dental implantların ve abutmantların üretiminde kullanılmış ve yüksek başarı oranı göstermiştir. Titanyumun siyah metalik parçalarının mukozadan yansıması ve yumuşak doku çekilmeleri sonrasında görünür hale gelmesi dezavantajlarıdır. ${ }^{2}$ Son dönemde bu dezavantajlarından dolayı titanyuma alternatif bir materyal olarak zirkonya da kullanılmaktadır. Zirkonya yüksek bükülme dayanımı ve sertlik gibi iyi mekanik özellikler gösterir ve uzun dönem yüklemelere dayanabilme kapasitesine sahiptir. ${ }^{44}$ Ayrıca titanyum implantlar ile karşılaştıılabilir osseöz bütünleşmeye ve metal yüzeylerden daha az plak akümülasyonuna sahiptir. ${ }^{2}$ Pirker ve Kocher klinikte uyguladıkları zirkonya dental implantlarda 5 yıl içinde herhangi bir kırık gözlemlemediklerini bildirmişlerdir. ${ }^{11,45}$

\section{Kişiye Özgü Kök Şekilli İmplantların Üretim Prosedürleri Zirkonya-kişiye özgü implantlar:}

Lokal anestezi altında diş çekimi, çekim soketine en az zararı verecek şekilde dikkatlice yapılır. Çekim soketi kürete edilerek temizlenir sonrasında salin solüsyonu ile irrigasyon yapılır ve iodoform emdirilmiş gazı bez yara yerine yerleştirilir. Çekilmiş olan diş kökü yüzeyi, interdental boşlukta kalmak şartıyla makroretansiyonlar veya tüm kök yüzeyine mikroretansiyonlar eklenmesiyle iki farklı şekilde modifiye edilebilir. ${ }^{12}$ İnce kortikal kemik çevresinde kırıma ve baskı kaynaklı kemik kaybını engellemek için implant çapı azaltııır. ${ }^{12}$ Zirkonya ile yapılmış kök şekilli immediat implantasyonunun stabilizasyonu ve osseointegrasyonunda, interdental boşluktaki makroretansiyonların ve ince kortikal kemik çevresinde implant

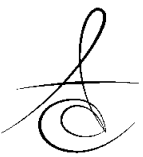


çapının azaltılmasının kritik rol aldığı kanıtlanmıştır. ${ }^{10,11,12}$ Yüzey modifikasyonunu takiben yüzey lazerle taranır, zirkonyum dioksit bloktan (özellikle yittrium ile stabilize edilmiş tetragonal polikristalin zirkonya) millenir. Tam olarak sinterlenmiş zirkonyanın frezelenmesi güç olacağı ve uzun süreler gerektireceğinden tam sinterlenmiş yerine kısmi sinterlenmiş veya önsinterlenmiş bloklar tercih edilmektedir. Milleme sonrasında kumlama ile yüzey pürüzlendirilir ve 8 saat boyunca sinterlenir. Çekimden 5 gün sonra iodoform emdirilmiş gazlı bez yara yerinden çıkarılır, alveolar soket tekrar kürete edilir ve salin solüsyonu ile yıkanır. İmplant ve dayanağın tek parça olduğu zirkonya implant çekim soketine yerleştirilir ve nazik vuruşlarla yerine yerleştirilir. Primer stabilite palpasyon ve perküsyon ile kontrol edilir. Hastadan 8 haftalık iyileşme periyodu sürecinde karşı tarafla çiğnemesi ve sert gıdalardan kaçınması istenir. Daimi restorasyon üç aylık iyileşme periyodunu takiben yapılır. Bu prosedürün dezavantajı diş çekimi ile implant yerleştirilmesi işlemlerinin ayrı cerrahi seanslarda yapılmasıdır.

\section{Titanyum-kişiye özgü implantlar:}

Son dönemde yeni bir yaklaşımla CBCT (Cone Beam Computed Tomography) kullanarak üç boyutlu model ve DLMF (Direct Laser Metal Forming) ile kök şekilli implant üretimi önerilmiştir. ${ }^{46,47} \mathrm{Bu}$ yöntem sayesinde ek bir cerrahi seans gereksinimi ortadan kalkmakta ve diş çekimi ile immediat implant yerleştirilmesi aynı seansta yapılmaktadır. Bir CBCT taramasında dişin görüntüsü işlenerek ve dönüştürülerek, diş çekimi öncesinde kökün aynısı olacak şekilde kök şekilli implant üretilebilir. ${ }^{46,47}$ Bu yöntemde diş çekimi öncesinde bir CBCT alınır ve elde edilen veri seti üç boyutlu model üretimi yazılımına aktarılır, yazılımda kök şekli simule edilir. Sanal kök stereolitografik (STL) dosya olarak izole edilir. İzole edilen STL dosyası tekrar 3D model üretimi yazılımına transfer edilir ve kökün çekim soketi ile uyumu kontrol edilir. Son olarak STL dosyası bilgisayar destekli tasarım yazılımına aktarıır, burada kök üzerine konik dayanak (abutment) dizayn edilir ve ince vestibuler kortikal kemik çevresinde implant çapı azaltılır. Tasarımı biten implant DMLF yöntemi ile üretilir. DLMF tekniği tabakalı üretime izin verir, böylece yüzeyde yüksek poröz, içte yüksek yoğunluklu yapılar oluşturulabilir. Bu şekildeki geçiş daha iyi yük adaptasyonuna, stresin azalmasına ve baskıya bağlı kemik kaybının azalmasına yardımcı olur. ${ }^{46,48-50}$ DLMF ile üretilen poröz yüzey, iyileşme sürecini hızlandırır ve osteointegrasyona olumlu katkı sağlar. ${ }^{46,48-50}$ Üretim sonrasında yüzey işlemleri uygulanır ve cerrahi aşamaya geçilir. Cerrahi işlem, zirkonya-kök şekilli kişiye özgü implantlardakine benzer şekilde devam eder.

Gelişen teknolojilerden daha çok yararlanarak uygulanan dental implantoloji diş hekimlerinin işlemlerini daha da kolaylaştırıp, endikasyon alanlarını da genişleterek daha başarılı sonuçlar almalarına yardımcı olabilecektir. Kişiye özgü implantlardan destek alınarak hazırlanacak protezlerde yine kişiye özgü dayanakların (abutment) kullanımının önemi artacaktır. Protetik sorunlara çözüm getirmek için önemli bir araç olan kişiye özgü dayanaklarla hazırlanmış protezlerin uzun dönem başarıları ise henüz bilinmemektedir.

\section{KAYNAKLAR}

1. Telleman G, Meijer HJ, Raghoebar GM. Long-term evaluation of hollow screw and hollow cylinder dental implants: clinical and radiographic results after 10 years. J Periodontol 2006;2:203-10.

2. Pirker W, Kocher A. True anatomical zirconia implants for molar replacement: a case report from an ongoing clinical study with a 2-year follow-up. J Oral Surg 2009;2:144-8.

3. Tolman DE, Keller EE. Endosseous implant placement immediately following dental extraction and alveoloplasty: preliminary report with 6-year follow-up. Int J Oral Maxillofac Implants 1991;6:24-8.

4. Koh RU, Rudek I,Wang HL. Immediate implant placement: positives and negatives. Implant Dent 2010;19:98-108.

5. Kazor CE, Al Shamari K, Sarment DP, Misch CE, Wang HL. Implant plastic surgery: a review and rationale. J Oral Implantol 2004;30:240-54.

6. Hodosh M, Povar M, Shklar G. The dental polymer implant concept. J Prosthet Dent 1969;22:371-80.

7. Lundgren $D$, Rylander $H$, Andersson $M$, Johansson C, Albrektsson T. Healing-in of root analogue titanium implants placed in extraction sockets. An experimental study in the beagle dog. Clin Oral Implants Res 1992;3:136-44.

8. Kohal RJ, Hurzeler MB, Mota LF, Klaus G, Caffesse RG, Strub JR. Custom-made root analogue titanium implants placed into extraction sockets. Clin Oral Implants Res 1997;8:386-92. 
9. Kohal RJ, Klaus G, Strubl JR. Clinical investigation of a new dental immediate implant system. The reimplant-system. Deutsch Zahn Z 2002;57:495-7.

10. Pirker W, Kocher A. Immediate, non-submerged, root-analogue zirconia implant in single tooth replacement. Int J Oral Maxillofac Surg 2008;37:293-5.

11. Pirker W, Kocher A. Immediate, non-submerged, root-analogue zirconia implants placed into singlerooted extraction sockets: 2-year follow-up of a clinical study. Int J Oral Maxillofac Surg 2009;38:1127-32.

12. Pirker W, Wiedemann D, Lidauer A, Kocher A. Immediate, single stage, truly anatomic zirconia implant in lower molar replacement: a case report with 2.5 years follow up. Int J Oral Maxillofac Surg 2011;40:212-6.

13. Regish KM, Sharma D, Prithviraj DR. An overview of immediate root analogue zirconia implants. ] Oral Implantol 2013;39:225-33.

14. Mangano C, Mangano F, Shibli JA, Luongo G, De Franco $M$, Briguglio $F$, Figliuzzi $M$, Eccellente $T$, Rapani C, Piombino M, Macchi A. Prospective clinical evaluation of 201 direct laser metal forming implants: results from a 1-year multicenter study on 62 patients. Lasers Med Sci 2012;27:181-9.

15. Chiapasco M, Casentini P, Zaniboni M. Bone augmentation procedures in implant dentistry. Int J Oral Maxillofac Implants 2009;24 Suppl:237-59.

16. Linkow LI. The blade vent-a new dimension in endosseous implantology. Dent Concepts 1968;11:3-12.

17. Linkow LI, Donath K, Lemons JE. Retrieval analyses of a blade implant after 231 months of clinical function. Implant Dent 1992;1:37-43.

18. Commissionat $Y$, Poulmaire F. Blade implants: new ideas. Rev Stomatol Chir Maxillofac 1996;97:2837.

19. Roberts RA. Types, uses, and evaluation of the plate-form implant. J Oral Implantol 1996;22:1118.

20. Veron C, Chanavaz M. Implant rehabilitation of distal mandibular atrophy using a blade implant. Rev Stomatol Chir Maxillofac 1997;98Suppl 1:1722.

21. Roberts R. Placement of plate-form implants using osteotomes. J Oral Implantol 2002;28:283-9.
22. Strecha J, Jurkovic R, Siebert T, Prachar P, Bartakova S. Fixed bicortical screw and blade implants as a non-standard solution to an edentulous (toothless) mandible. Int J Oral Sci 2010;2:105-10.

23. Trisi $P$, Emanuelli $M$, Quaranta $M$, Piattelli A. A light microscopy, scanning electron microscopy and laser scanning microscopy analysis of retrieved blade implants after 7 to 20 years of clinical function. J Periodontol 1993;64:374-8.

24. Proussaefs $P$, Lozada J. Evaluation of two Vitallium bladeform implants retrieved after 13 and 21 years of function: a clinical report. J Prosthet Dent 2002;87:412-5.

25. Di Stefano D, Iezzi G, Scarano A, Perrotti V, Piattelli A. Immediately loaded blade implant retrieved from a man after a 20- year loading period: a histologic and histomorphometric case report. J Oral Implantol 32:171-6.

26. Aljateeli M, Wang HL. Implant microdesigns and their impact on osseointegration. Implant Dent 2013;22:127-32.

27. Shalabi MM, Gortemaker A, Van'tHof MA, Jansen $\mathrm{JA}$, Creugers $\mathrm{NH}$. Implant surface roughness and bone healing: a systematic review. J of Dent Res 2006;85:496-500.

28. Monjo M, Petzold C, Ramis JM, Lyngstadaas SP, Ellingsen JE. In vitro osteogenic properties of two dental implant surface. Int $\mathrm{J}$ of Biomater 2012;2012:181024.

29. Elias CN, Gravina PA, Silva Filho CE, Nascente P. A. Preparation of bioactive titanium surfaces via fluoride and fibronectin retention. Int J of Biomater 2012;2012:290179.

30. Mangano C, Piattelli A, Mangano F, Perrotti V, Iezzi G. Immediate loading of modified acid etched dental implants in postextraction sockets: a histological and histomorphometrical comparative study in nonhuman primate papio ursinus. Implant Dent 2009;18:142-50.

31. Sesma N, Pannuti C, Cardaropoli G. Retrospective clinical study of 988 dual acid-etched implants placed in grafted and native bone for single-tooth replacement. Int J Oral and Maxillofac Implants 2012;27:1243-8.

32. Choi JY, Lee HJ, Jang JU, Yeo IS. Comparison between bioactive fluoride modified and bioinert anodically oxidized implant surfaces in early bone

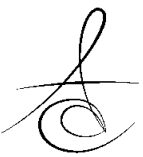


response using rabbit tibia model. Implant Dent 2012;21:124-8.

33. Degidi M, Nardi D, Piattelli A. 10-year follow-up of immediately loaded implants with TiUnite porous anodized surface. Clin Implant Dent and Relat Res 2012;14:828-38.

34. Mendes VC, Moineddin R, Davies JE. The effect of discrete calcium phosphate nanocrystals on bonebonding to titanium surfaces. Biomaterials 2007;28:4748-55.

35. Ryan G, Pandit A, Apatsidis DP. Fabrication methods of porous metals for use in orthopaedic applications Biomaterials 2006;27:2651-70.

36. Li JP, Li SH, Van Blitterswijk CA, de Groot K. Anovel porous Ti6A14V: characterization and cell attachment. J of Biomed Mater Res A 2005;73: 223-33.

37. Traini T, Mangano C, Sammons RL, Mangano $F$, Macchi A, Piattelli A. Direct laser metal sintering as a new approach to fabrication of an isoelastic functionally graded material for manufacture of porous titanium dental implants. Dent Mater 2008;24:1525-33

38. Shibli JA, Mangano C, D'Avila S, et al. Influence of direct laser fabrication implant topography on type IV bone: a histomorphometric study in humans. J of Biomed Mater Res A 2010;93:607-14.

39. Mullen L, Stamp RC, Brooks WK, Jones E, Sutcliffe C]. Selective laser melting: a regular unit cell approach for the manufacture of porous, titanium, bone in-growth constructs, suitable for orthopedic applications. J of Biomed Mater Res B Appl Biomater 2009;89:325-34.

40. Stamp R, Fox P, O'Neill W, Jones E, Sutcliffe C. The development of a scanning strategy for the manufacture of porous biomaterials by selective laser melting. J of Mater Sci Mater Med 2009;20:1839-48.

41. Hollander DA, von Walter $M$, Wirtz $T$, et al. Structural, mechanical and in vitro characterization of individually structured Ti-6Al-4V produced by direct laser forming. Biomaterials 2006;27:955-63.

42. Mangano C, Raspanti M, Traini T, Piattelli A, Sammons R. Stereo imaging and cytocompatibility of a model dental implant surface formed by direct laser fabrication. J of Biomed Mater Res A 2009;88:823-31.
43. Dabrowski B, Swieszkowski W, Godlinski D, Kurzydlowski KJ. Highly porous titanium scaffolds for orthopaedic applications. J of Biomed Mater Res B Appl Biomater 2010;95:53-61.

44. Silva VV, Lameiras FS, Lobato ZI. Biological reactivity of zirconia-hydroxyapatite composites. J Biomed Mater Res 2002;63:583-90.

45. Oliva J, Oliva X, Oliva DJ. One-year follow-up of first consecutive 100 zirconia dental implants in humans. a comparison of two different rough surfaces. Int J Oral Maxillofac Implants 2007;22:430-5.

46. Mangano FG, Cirotti B, Sammons RL, Mangano C. Custom-Made, Root-analogue direct laser metal forming implant: a case report. Lasers Med Sci 2012;27:1241-5.

47. Moin DA, Hassan B, Mercelis P, Wismeijer D. Designing a novel dental root analogue implant using cone beam computed tomography and cad/cam technology. Clin Oral Implants Res 2013;24 Suppl A100:25-7.

48. Mangano C, Mangano F, Shibli JA, Luongo G, De Franco $M$, Briguglio $F$, et al. Prospective clinical evaluation of 201 direct laser metal forming implants: results from a 1-year multicenter study. Lasers Med Sci 2012;27:181-9.

49. Mangano C, Piattelli A, Raspanti M, Mangano F, Cassoni A, Iezzi $G$, et al. Scanning electron microscopy (sem) and x-ray dispersive spectrometry evaluation of direct laser metal sintering surface and human bone interface: a case series. Lasers Med Sci 2011;26:133-8.

50. Mangano C, Mangano FG, Shibli JA, Ricci M, Perrotti V, d'Avila $S$, et al. Immediate loading of mandibular overdentures supported by unsplinted direct laser metal-forming implants: results from a 1-year prospective study. J Periodontol 2012; 83:70-8.
Yazışma Adresi
Doç Dr Bora Bağış
İzmir Katip Çelebi Üniversitesi
Diş Hekimliği Fakültesi
Protetik Diş Tedavisi AD
35640 Çiğli-İzmir
Telefon: 02323254040
E-mail: bbagis@yahoo.com 